\title{
Hybrid Polymeric Nanocomposites Based High Performance Oleds: A Review
}

\author{
R. Kandulna ${ }^{4 *}$, \\ U. Das ${ }^{1}$, \\ Rimpi ${ }^{1}$, \\ B. Kachhap ${ }^{3}$, \\ N. Prasad ${ }^{2}$
}

\begin{abstract}
This review reports the recent significant progress for achieving the synthesis of various types of polymer-based nanocomposites and understanding of the basic principle which determine their optical, electronic and magnetic properties. Some of the polymeric nanocomposite materials show remarkable electrical as well as optical properties regarding towards interest for applications in OLEDs. Nanoparticles which are basically inorganic, plays important role in the opto-electronic field. A nanoparticle with conjugate polymer matrix makes polymer nanocomposite and that nanocomposite used as an active emissive layer between the structures of optoelectronic device like OLEDs, OPVs etc. Application of such polymeric nanocomposite materials are discussed in this present communication with their success and failure, towards their optical as well as electrical properties in the fabrication of OLEDs.
\end{abstract}

Keywords : Nanocomposites, OLEDs structure, hybrid materials, opticalelectrical properties, lifetime \& device stability.

Corresponding author: 'Department of Physics YBN University, Ranchi, email:rohitkandulna4@gmail.com

Authors: ${ }^{2}$ Department of Mathematics YBN University, Ranchi

${ }^{3}$ University Department of Botany, Ranchi University, Ranchi.

${ }^{4}$ University Department of Physics, DSPM University, Ranchi

\section{Introduction}

The word nanocomposite is used for those composite or hybrid material for which size of one component is in the nanometre range $\left(1 \mathrm{~nm}=10^{-}\right.$ 
$9 \mathrm{~m}$ ) [1]. Nanosized materials show interesting physical properties which lead to unexpected performance for OLED applications. It makes them fairly attractive for optoelectronics [2]. In general, a nanocomposite bears enhanced properties in comparison with the individual components, which allow its use in a particular application. Such nanocomposite should be able to replace more than one material, which should require for obtaining similar properties for desired application. Polymer nanocomposites made of polymers and inorganic nanoparticles are markedly used for automotive applications due to their excellent mechanical properties, low cost and easy transferable [3]. The behaviour of such nanocomposites is mostly dependent on the design parameters such as concentration, structure, size and shape etc. The above said parameters lead to unexpected growth in properties and performance towards electronics application also. Due to these reasons, preparation of nanocomposites seeks significant role in obtaining high quality materials with improved efficiency. The current trend in polymeric nanocomposites with enhanced optical and electronic properties. Active elements in organic light emitting diodes using organic materials have paved the path for the possible replacement of replacement of inorganic counterparts in several electronic applications. Organic optoelectronics devices take better advantage of organic materials than inorganic materials due to ease of processing, light weight, large surface area, flexibility and low cost [4]. Organic devices failure even at ambient temperature by the use of organic nanoparticle, so for enhancement of the stability as compared the inorganic phase into the polymer matrix is attributed to the strong interfacial interactions between the polymer and inorganic fillers [5]. However, the durability of organic devices is still short or than that of conventional semiconductors due to the above discussed drawbacks. Further there is an additional advantage of the use of polymer nanocomposites in optoelectronics devices like OLEDs and i.e., the formation of pathways for carrier transport. It improves the electrical conductivity of the films by the use of polymeric nanocomposites as active materials in OLEDs [6]. It is expected to substantially improve their performance as well as their life time of the optoelectronic devices.

\section{Utilization of Polymers in OLEDs}

Polymer organic light emitting diodes (OLEDs) are quite efficient and require a relatively small amount of the light produced [7]. Device efficiencies can be improved by selecting appropriate combination of polymer and device modifications. The light emitting polymer (LEP) can be modified to increase 
its photoluminescence and quantum yield (PLQY) and while devices can be improved by adding a thin polymeric layer between the hole transport layer and LEP. This additional layer is commonly referred to as an interlayer/primer layer (IL).

The LEP can be designed to exhibit higher electron mobility than hole mobility [8]. On the other hand, the interlayer/primer layer is designed with hole transport possesses a higher hole mobility than electron mobility, i.e., electron transport is favoured in the LEP, while hole transport is favoured in the primer layer. The net effect is that electron and hole charges accumulate at the LEP: IL interface and away from either the cathode or anode. In this way, charge balance within the devices can be obtained, exciton (electron-hole pair in the excited state) formation can be maximized, and exciton quenching by either cathode or anode can be avoided.

The major class of polymers that are used in OLEDs have been shown below along with its chemical structures as shown in Fig.1.

- PMMA (Poly methyl methacrylate)

- PPP (Poly p- phenylene)

- PEDOT (Poly ethylenedioxythiophene)

- PVA (Poly vinyl alcohol)

- PANI (Polyaniline)

- PPV (Poly phenylene vinylene)

- PFO (Polyfluorene)

- PT (Polythiophene) 


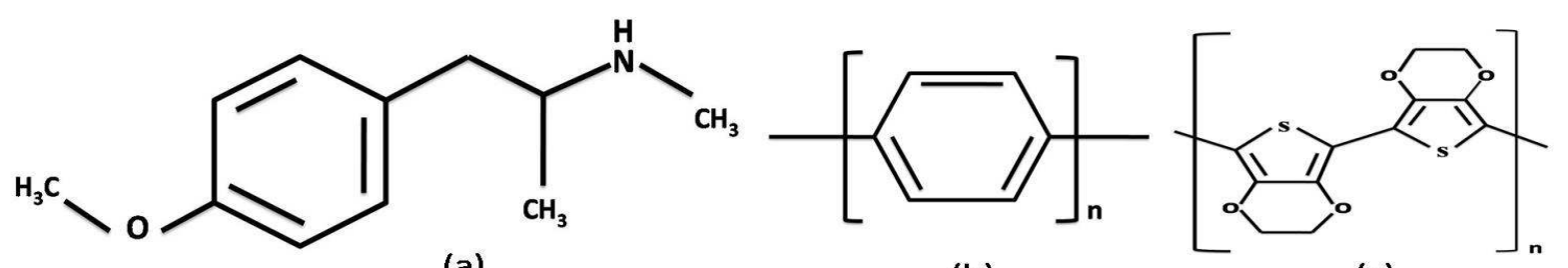

(a)

(b)

(c)

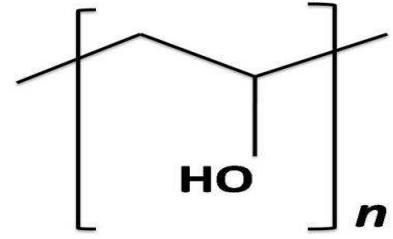

(d)

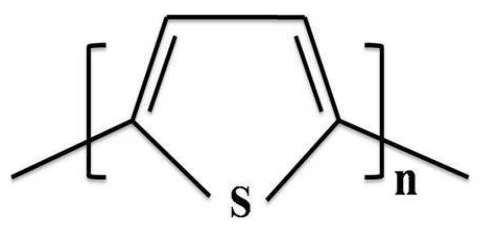

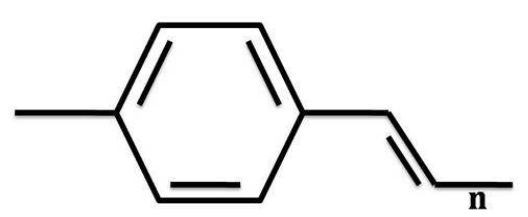

(e)

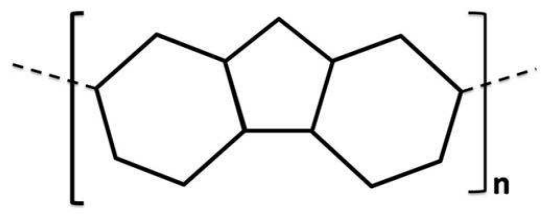

(f)

Fig.1- Chemical structures of (a) PMMA, (b) PPP, (c) PEDOT, (d) PVA, (e) PANI, (f) PPV, (g) PFO, (h) PTP.

\section{Premier Hybrid-Nanocomposites Classifications for OLEDs}

In the recent year due to emerging trends of organic light emitting diode, the process of strengthening polymers using fillers of inorganic or organic. In organic origin has been popularised significantly. It is common employed for in the production of modern plastics and is typically used in the field of optoelectronic industry. Polymeric nanocomposites can be formed by adding metallic, semiconducting and dielectric nanocrystal into polymer matrix for enhancing the efficiency and durability of the optoelectronic devices. By using of inorganic nanoparticles in the OLED applications, it is seen that, it influences properties of the pure polymer. The literature search composites for OLEDs show that electrical and spectroscopic properties of the conjugated polymers can be enhanced by incorporation of nanocomposite $\mathrm{TiO}_{2}$ in the polymers [9]. Polymeric nanocomposites followed by its properties lead to the controlled particle size distribution, dispersion as well as interfacial interactions. The main objective is to generate bright light by interacting electrons and photons in devices for OLED applications.

\subsection{Classification of polymeric nanocomposites}


Polymeric nanocomposites can be broadly classified as per the following

- Clay composites

- Carbon nanotube composites

- Nanofiber composites

- Inorganic particle composites.

\subsubsection{Clay nanocomposites}

Nanoclays are nanoparticles of layered mineral silicates. In other words, these are inorganic particles without any definite composition or crystallinity. The clay mineral (also called a phyllosilicate) is usually a fraction of hydrous, magnesium, or aluminium silicates. Basically, clay mineral in nature contains two types of sheets i.e., tetrahedral (T) and octahedral (O). Depending on chemical composition, nanoparticle morphology is organized into several classes such as montmorillonite, bentonite, kaolinite, hectorite and halloysite. Plate like montmorillonite is the most common nanoclay used in materials applications due to high surface area and high reactivity.

Polymer-clay nanocomposites have been used as active components in organic optoelectronic devices like OLEDs, OPVs etc. These are inorganic layered materials with unique physical properties. As per the commercial protocol of novel optoelectronics devices, the permeability of oxygen and water vapour must be controlled. As a result, the device stability and lifetime could be improved. Clays are necessarily needed to be treated in order to interface with the organic matrices to form a polymer nanocomposite structure. In such nanoclay composite, the polymer chains are inserted in between the clay layers and their collections is reduced. The single clay layer influences the behaviour of the devices lowering current densities and increasing light output in comparison with pure polymer systems [10]. By inserting nanocomposite, a pure light emission can be obtained and energy site (excimer) formation can be efficiently eliminated.

\subsubsection{Carbon nanotube composites}

For the detailed study of inorganic electronics, carbon nanotubes offer numerous advantages such as high current carrying capacity, ballistic 
transport and absence of dangling bonds etc. However, in the case of organic electronics, carbon nanotubes offer slightly difference advantages such as high conductivity, high carrier mobility, optical transparency, flexibility, robustness and environmental resistance etc [11-12]. Because of these reasons they are seriously consider as being considered as contenders to silicon and ITO. The origin of carbon nanotubes in the field of organic electronics (OLEDs, OPVs etc.) includes applications of multiwall- carbon nanotubes ( $M W C N T_{S}$ ) and single-wall carbon nanotubes (SWCNTS) because of their better satisfactory results [13-14]. Applications of carbon nanotubes as hole-transport layers, as electron transport layers and as transparent electrodes in organic light emitting diodes have been discussed and daunting challenges are brought into the limelight. For improving the electrical transport in the active composite layer, it needs to incorporate derivatives of conjugate polymers with low concentration carbon nanotubes. The small concentration of CNTs causes to enhance the electrical properties such as mobility of minority carriers thereby producing a balance of mobility. As a result, it causes to improve the optical properties and efficiency accordingly.

\subsubsection{Nanofiber composites}

Carbon nanofibers (CNFs) vapour grown carbon fibres (VGCFs) and vapour grown carbon nanofibers (VGCNFs) are cylindrical nanostructures with graphene layers arranged as stacked cones, cups or plates. In fact, carbon nanofibers with graphene layers wrapped into perfect cylinders are called carbon nanotubes. Generally, carbon nanofibers (CNFs) are a unique form of vapour grown carbon fibres that fill the gap in physical properties between conventional carbon fibres and carbon nanotubes. These nanofibers provide a larger surface area with surface functionalisation in the fibre by reducing its diameter. These $\mathrm{CNFs}$ are not concentric cylinders in structure. The cylindrical fibre varies in its length from $100 \mu \mathrm{m}$ to several $\mathrm{cm}$ and its diameter varies from 100 to $200 \mathrm{CNFs}$ are generally truncated in structured, but there are wide range of morphologies. CNFs have the morphology where these are hollow at the centre and have a larger diameter than MWNT but the individual layers are not arranged in concentric tubes.

It is a challenge to use them on nanometre scale in order to fully exploit their flexibility and potential. Aggregation of conjugate-based nanofibers to electrode makes the probing of their electrical properties possible and it represents an important step for the realization of nanoscale OLED. In the recent years, different ideas to achieve such contacts have been proposed, 
either by defining the area of the electrical contacts or by confining the size of active materials in the field of electronics devices (OLED). There are some silica nanoparticles to process different nanocomposites with different resin systems for high-temperature applications and it can be used for optoelectronics applications. It has been seen that poor creep resistance and dimensional stability have been improved by adding $\mathrm{TiO}_{2}$ in polyamide thermoplastic composites. An equal amount of loading, nanoparticle infusion brings superior thermal and mechanical properties than micro sized particlebased polymer composites.

\subsubsection{Inorganic particle composites.}

Inorganic particle composites are mostly used in the different field of electronic devices for getting better and desired performance. In order to improving the different properties of the nanocomposite's materials, nanoparticles can be developed from different organic-inorganic origin. Nanoparticles can be developed from metals and be used as stable composite cathodes (Ca/Al, Mg/Ag, Mg/MgAg, Gd/Al etc.) in OLEDs [15]. Also, metal oxides and non-metal oxides play important role towards OLEDs applications. Nanoparticles are selected based upon different properties like optical, electrical and thermal one in OLEDs application [16]. Further inorganic particles are used in composite for getting better stability and high efficiency. Since OLEDs are attractive and easy transferable due to high efficiency and light weight. Hence these used not only in OLEDs but also in many other opto- electronics applications. In photo voltaic cells, organic particles are used as active layers. In the field of opto-electronic industry, its use is increased day by day and will develop organic devices with better performance. For the distance future, it may be predicted that tailoring of spontaneous emission properties using nanostructured environments will become standard if it is allowed for direct tunnelling of the emitted photons into the desired modes.

\section{Applications of Hybrid Nanocomposite Materials for OLEDs}

In recent years, there has been increasing interest in combining the nanotechnology advanced with organic devices such as OLEDs. Nanocomposite material should take advantages from components used in optical and electrical applications. The use of conjugated polymers is required for optoelectronics applications. Such conjugated polymers present a large range of band gap values and it covers almost all the range of all visible 
spectrums. It is observed that these conjugated polymers with nanocomposite shows a better electrical conductivity, enabling their use in electronic devices such as OLEDs, solar cells etc. Polymer nanocomposite have many important roles to play for enhancing the properties in OLEDs in addition to their primary role to enhance some specific characteristic. Polymer nanoparticles are also expected to increase the stability of the host material. Polymer's nanocomposites are sensitive to atmosphere as well as light exposure. Conjugate polymers can rapidly degrade by oxidation and photo-oxidation. In order to prevent them from premature degradation, an organic device must be protected against air and water by encapsulation.

\subsection{OLED Structures}

The basic OLED structure consist of several layers as discussed below

1. Electron Transport Layer (ETL)

2. Hole Transport Layer (HTL)

3. Emissive Layer

4. Substrates

5. Cathode

6. Anode

In the beginning, the most basic polymer OLEDs consisted of a single organic layer and it has been shown in the Figure. In modern OLEDs incorporates a simple bi-layer or double layer structure, consisting of electron transport layer (ETL) and hole transport layer (HTL). More recent developments in OLED architecture improve efficiency such as quantum efficiency. In the graded hetero-junction architecture, the composition of hole and electron transport materials varies continuously within the emissive layer with a dopant emitter and it may be triple layer or multi-layer. OLEDs with single layer, double layer, triple layer and multi-layer have been shown in Fig.2 


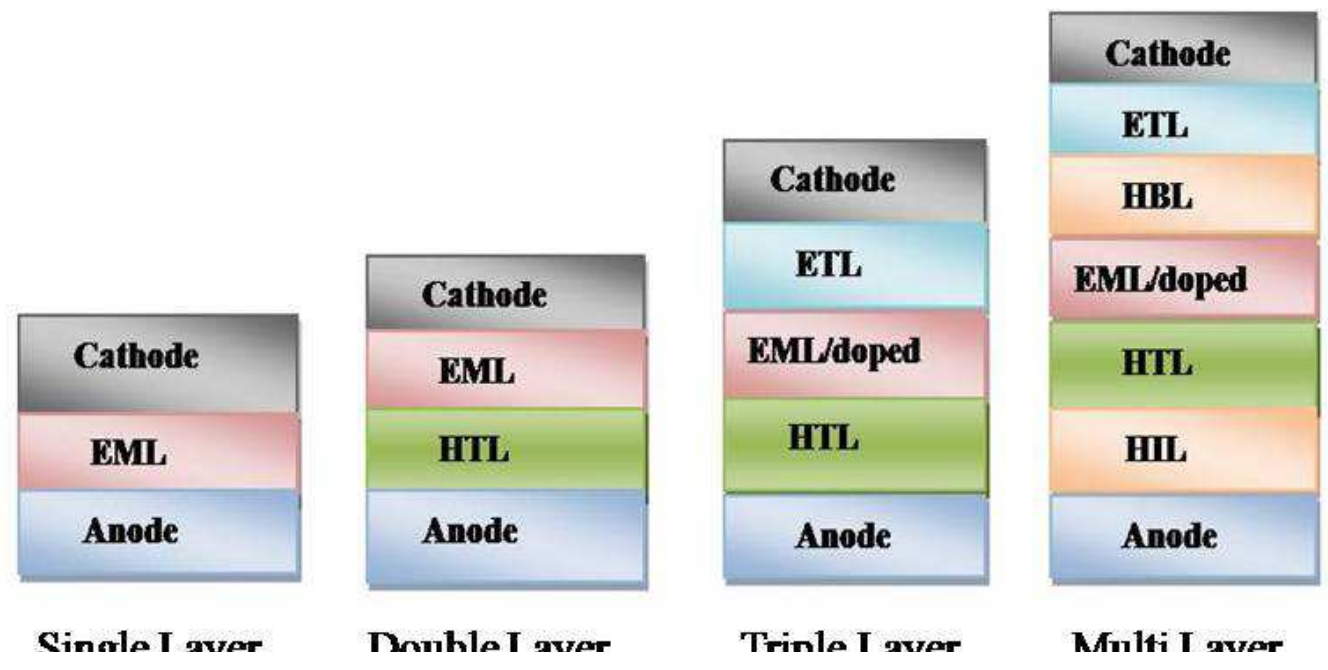

layers

Fig.2- Schematic illustration for OLED structures with different

\subsubsection{HOMO-LUMO Interaction}

Every molecule contains its molecular orbital. Highest occupied molecular orbital (HOMO) and a lowest unoccupied molecular orbital (LUMO) are two important orbitals which can be found in any molecule.

There is sensible energy match between HOMO and LUMO, so that there can be substantial lowering in energy. If this is enough to overcome the repulsion from interaction of other OMOs (occupied molecular orbital) with one another, a reaction occurs. The favourable condition of performing the reaction is the necessity of high HOMO in one reaction associate and law LUMO in another side. Every molecule has specific functional group. Using this functional group, it is anticipated that molecule should have usually high HOMO's and it should have usually law LUMO as well as it also determines that which functional group should react with each other. Fig. 3 shows the HOMO-LUMO interaction in OLED structure with different component. 


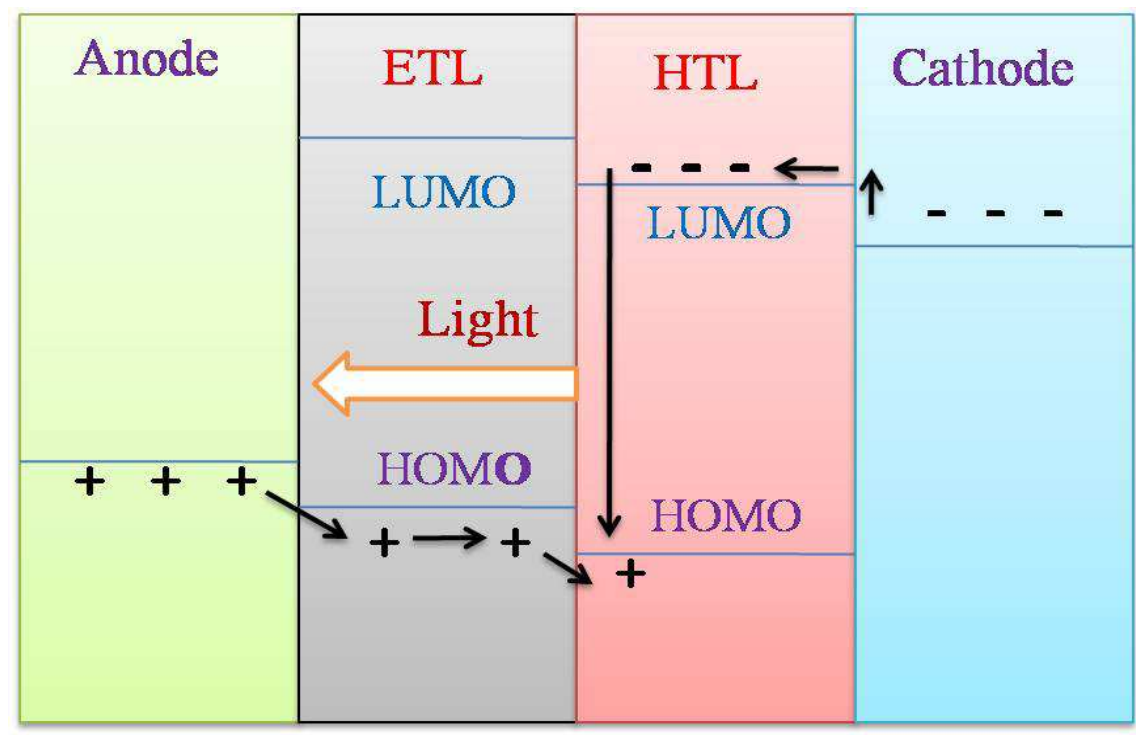

Fig.3: HOMO -LUMO interaction in OLED structure

\subsubsection{Materials used for different layers}

The literature survey for organic optoelectronics devices, reveals that the materials used in OLEDs need to have good thermal stability, low turn-on voltages, high conductivity, high electroluminescence, long durability and good operational stability. The role of organic materials as active components can be realised by the necessity of optimizing the characteristics of the devices. Depending on the requirement of different layers of OLEDs organic materials can be employed as per following;

(I) Materials for cathode

Cathode materials used for OLEDs should possess low work function, low threshold voltage and they must be efficient electron injectors. The effective cathode materials such as $\mathrm{Ca}, \mathrm{K}$ and $\mathrm{Li}$ infer that they exhibit poor corrosion resistance and high chemical reactivity with the organic medium. Metal alloys with low work functions like $\mathrm{Mg}-\mathrm{Ag}$ and $\mathrm{Al}-\mathrm{Li}$ are usually used for cathodes. With $\mathrm{Al}$-based composite cathodes of $\mathrm{Al}-\mathrm{LiF}$ or $\mathrm{Al}-\mathrm{CsF}$, undoped organic bilayer devices, based on $\mathrm{Alq}_{3}$ as the light emitting medium and TDP as the hole transport layer, showed better luminance efficiency [17]. By inserting a layer of $\mathrm{LiF}$ or $\mathrm{Al} / \mathrm{LiF}$ cathode with appropriate amount in OLEDs devices, have been enhanced the electroluminescent performance with 
luminance greater than $20,000 \mathrm{~cd} / \mathrm{m}^{2}$ [18]. The work function of cathode metals is depicted in Table 1.

Table 1- Work functions for various cathode metals used in OLED [19]

\begin{tabular}{cr}
\hline Element & Work funct \\
$\mathrm{Al}$ & 4.30 \\
$\mathrm{Ca}$ & 3.00 \\
$\mathrm{Li}$ & 2.95 \\
$\mathrm{Sm}$ & 2.73 \\
$\mathrm{Mg}$ & 3.70 \\
$\mathrm{Yb}$ & 2.63 \\
$\mathrm{Ag}$ & 4.32 \\
$\mathrm{Zn}$ & 4.47 \\
$\mathrm{Cu}$ & 4.70
\end{tabular}

(III) Materials for Hole Transport Layer (HTL)

Hole-transporting materials are those that accept hole carriers with a positive charge and transport them. Therefore, materials which have low ionization potentials together with low electron affinities usually function as hole transporting materials. In other words, charge-transporting materials with donating electron-donating properties usually serve as hole-transporting materials. In addition to the role of charge transport, hole transporting materials used in OLEDs play a role facilitating hole injection from the anode, into the emitting layer by a stepwise process. Hole transporting materials also play the role of blocking electrons from escaping from the emitting layer in OLEDs.

(IV) Materials for Anode

The materials are used in OLEDs as anode must be good transparency, good conductivity, high chemical stability and high work function also. Recent developments in organic electronics such as organic 
light emitting diodes (OLEDs), indium tin oxide (ITO) is the suited anode materials for this device. The work function of the ITO is the order of $4.6 \mathrm{Ev}$. Transparent conductive oxide (TCO) based materials such as impurity doped ZnO films e.g., AZO, GZO and ZZO have been used as non-ITO anodes and deposited on glass substrates by pulsed laser deposition and characterized as a function of oxygen background gas pressure [20]. It gives better luminance power efficiency and enhances light output also. Some of the other TCO materials such as gallium-indium-oxide (GIO), gallium-indium-tin oxide (GITO), zinc-indium-oxide (ZIO), zinc-indium-tin oxide (ZITO) have been used as effective OLED anodes and exhibited high electrical conductivity, outstanding optical transparency and work functions considerably greater than that of commercial ITO [21].

(VI) Materials for Electron Transport Layer (ETL)

Like hole transport layer, the electron transport-layer is used attaining efficient electron injection, transport, and recombination in LEDs from the metal cathode. Metal cathodes are chosen in OLEDs which have low work-functions e.g., calcium, magnesium, aluminium etc. Different classes of electron transport materials used to enhance the performance of small molecule and polymer-based OLEDs and performance like quantum efficiency, brightness, luminance yield, turn-on or drive voltage depend on many extrinsic variables such as thicknesses of the layers. Like HTL electron transport layer in OLEDs play a role in hole blocking as well as acceptance and transport of electrons. A very few useful ETMs (Electron Transport Materials) with superior electron mobility have been developed. The most widely used ETMs e.g., Oxadiazole is an organic material with good electrontransport properties. The first used as an electron transport material in a bilayer OLED based on a triphenylamine derivative as the emissive material was oxadiazole molecule 2-(4-biphenyl)-5-(4-tert-butylphenyl)-1, 3, 4oxadiazole (PBD, 3), with an electron affinity of $2.16 \mathrm{eV}$ and an ionization potential of $6.06 \mathrm{eV}$. The properties of some material for electron transport materials are depicted in Table 2. It is found that Common electron-transport materials $\mathrm{Alq}_{3}$ has been subsequently implemented in all coated blue fluorescent OLEDs as smooth and uniform film [22].

Table 2- Properties (physical, chemical and optical) of some Electron Transport materials [45]. 


\begin{tabular}{|c|c|c|c|c|c|}
\hline \multirow{2}{*}{ Materials } & & Molecular & $\begin{array}{l}\text { TGA } \\
(0.5 \%\end{array}$ & Absorption & Emission \\
\hline & $\begin{array}{l}\text { Formul } \\
\text { a }\end{array}$ & & $\begin{array}{l}\text { weight } \\
\text { loss) }\end{array}$ & $\begin{array}{l}\text { wavelength(nm } \\
\text { ) }\end{array}$ & $\begin{array}{l}\text { wavelength(n } \\
\text { m) }\end{array}$ \\
\hline PBD & $\mathrm{C}_{24} \mathrm{H}_{22} \mathrm{~N}$ & 354.44 & $>210^{\circ} \mathrm{C}$ & $305 \mathrm{~nm}$ & $364,380 \mathrm{~nm}$ \\
\hline
\end{tabular}

\begin{tabular}{|c|c|c|c|c|c|}
\hline Liq & $\begin{array}{l}\mathrm{C}_{9} \mathrm{H}_{6} \mathrm{Li} \\
\mathrm{NO}\end{array}$ & 151.09 & $>310^{\circ} \mathrm{C}$ & $261 \mathrm{~nm}$ & $331 \mathrm{~nm}$ \\
\hline Bphen & $\mathrm{C}_{24} \mathrm{H}_{16} \mathrm{~N}$ & 332.4 & $>240^{\circ} \mathrm{C}$ & $272 \mathrm{~nm}$ & $379 \mathrm{~nm}$ \\
\hline TAZ & $\mathrm{C}_{30} \mathrm{H}_{27} \mathrm{~N}$ & 429.56 & $>250^{\circ} \mathrm{C}$ & $290 \mathrm{~nm}$ & $370 \mathrm{~nm}$ \\
\hline BAlq & $\begin{array}{l}\mathrm{C}_{32} \mathrm{H}_{25} \\
\mathrm{AlN}_{2} \mathrm{O}_{3}\end{array}$ & 512.53 & $>230^{\circ} \mathrm{C}$ & $259 \mathrm{~nm}$ & $334,477 \mathrm{~nm}$ \\
\hline TPBi & $\mathrm{C}_{45} \mathrm{H}_{30} \mathrm{~N}$ & 654.76 & $>350^{\circ} \mathrm{C}$ & $305 \mathrm{~nm}$ & $359,370 \mathrm{~nm}$ \\
\hline $\mathrm{BCP}$ & 6 & 360.45 & $>240$ & $277 \mathrm{~nm}$ & $386 \mathrm{~nm}$ \\
\hline
\end{tabular}

PBD-2-(4-Biphenyl)-5-(4-tert-butylphenyl)-1,3,4- oxadiazole, $\quad$ Liq- $^{-} \quad$ 8- $^{-}$ 
Hydroxyquinolinolato-lithium, Bphen- 4,7-Diphenyl-1,10-phenanthroline, TAZ- 3-(4-Biphenyl)-4-phenyl-5-tert-butylphenyl-1,2,4-triazole,BAlq-Bis(2methyl-8-quinolinolate)-(phenylphenolato)aluminium, TPBi- 2,20,2"-(1,3,5Benzinetriyl)-tris(1-phenyl-1- H-benzimidazole) BCP- 2,9-Dimethyl-4,7diphenyl-1,10- phenanthroline, TGA- Thermogravimetric analysis

(VII) Materials for Emissive Layer (EL)

The advantages of organic materials over inorganic materials are their colour gamut and high fluorescence efficiency. Light is produced in organic materials by the fast decay of excited molecular states, and the colour of light depends on the energy difference between those excited states and molecular ground level. There are three different types of emissive materials classified with high efficiency, increasing lifetime and colour purity for OLEDs. These emissive materials are such as small molecules, polymers and dendrimers. Classification of emissive materials is shown below in Fig.4 with appropriate diagram.

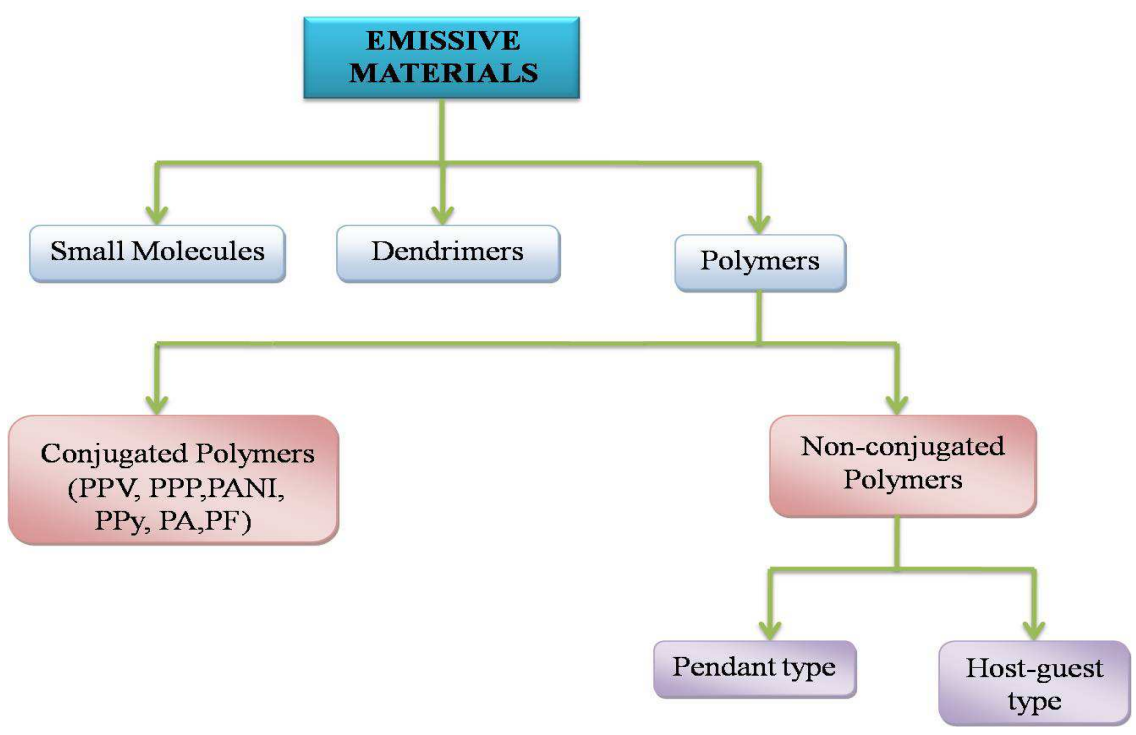

Fig.4- Diagram showing the classification of emissive materials

(VIII) Substrates

Substrates play important role in the field of opto-electronics devices, particularly lighting applications. There are a number of requirements for OLED substrates to meet in order to ensure cost, lifetime, efficiency, and ease of processing. Optical properties are one of the specific requirements and in 
OLEDs it should optically clear substrate or optically transparent. Low surface roughness and asperities over short distance must be avoided. The substrate should not release contaminants and should be inert against process chemicals for OLED application. The other properties like electrical and magnetic properties, thermal and thermo-mechanical properties also play key role in the OLEDs application. It is seen in the recent developments these properties serve as guideline and may vary depending on the application and device fabrication methodology. The various substrate material and its properties are depicted in Table 3.

Table 3- Substrate materials and their properties in OLED [23].

\begin{tabular}{llcccc}
\hline Substrate & MPT( $\left.{ }^{\circ} \mathrm{C}\right)$ & VT(\%) & CTE & $\begin{array}{c}\text { Tg/melting } \\
\text { point( }\left({ }^{\circ}\right)\end{array}$ & TC(W/mk) \\
\hline PI & 200 & Orange & $30-60$ & 400 & 0.5 \\
PES & 200 & 90 & $15-55$ & 185 & $\sim 0.2$ \\
PEN & 150 & 87 & 13 & 120 & $\sim 0.1$ \\
PET & 120 & 89 & 15 & 260 & $\sim 0.2$ \\
PC & 200 & 90 & $60-70$ & 150 & $\sim 0.2$ \\
Paper & Low & Var. & Var. & Var. & Var. \\
Stainless & 900 & & $10-18$ & 1400 & $>16$ \\
steel & 350 & Opaque & $\sim 23$ & 660 & $>200$ \\
Aluminum & $200-600$ & Opaque & $3-9$ & $>500\left(\mathrm{~T}_{\mathrm{g}}\right)$ & $\sim 1.0$ \\
Glass & & & & &
\end{tabular}

92

Where, MPT- Maximum Processing Temperature. CTE- coefficient of thermal expansion, VT-Visible Transmisson, TC- Thermal Conductivity, PIPolyimide, PES-Polyether-sulfone, PEN- Polyethylene naphthalate, PETPolyethylene terephthalate, PC- Polycarbonate 


\section{Conclusion}

There are large numbers of issues identified for the technology and commercialization of organic optoelectronics devices. The main target is to increase the lifetime of these devices with hard working without failure by using composite materials. In spite of deep investigations towards organic materials, basically the degradation mechanisms have not been briefly understood yet. For improving the stability of polymers, people use nanocomposite materials taking as an alternative approach. These nanocomposite materials develop covalent bonding to the organic counterpart and making stronger their structure in the OLED application. The high performance in efficiency development and durability of devices are usually found improving with composite materials. Contribution of inorganic part in making OLED enhances the stability of the composite as compared to the organic parts. Recent development in research towards organic devices results that the presence of inorganic nanoparticles in an organic host matrix which tends to heal existing defects in the material. In the field of OLEDs, hybrid materials are useful and these are more efficient for replacing polymers in different parts of the devices such as transporting layer, transparent electrode, and active layer. Hybrid materials also provided that the concentration of the inorganic materials does not change in the optical properties of the corresponding film. By the use of hydride materials, the performance and lifetime organic devices are usually found improving and there is some probability a loss in interesting properties of the organic materials.

At present time nanotechnology is progressing very rapidly and this progress will help the field of organic light emitting diode development. I think there will be big hope developing towards opto-electronics devices that the issues in fabricating hybrid materials by which the earth will become more luminous with the brightest lightning in the near future.

\section{Acknowledgement}

The authors are thankful to the anonymous referees of the journal for their handy suggestions to improve the quality of the article.

\section{Declaration of Conflict of Interest}

The authors declared no potential conflicts of interest with respect to the research, authorship, and/or publication of this article. 


\section{Funding}

The author received no financial support for the research, authorship, and/or publication of this article.

\section{References}

[1] Camargo, P.H.C.; Satyanarayana, K.G.; Wypych, F. Nanocomposites: synthesis, structure, properties and new application opportunities. Materials Research 12, no. 1 (2009): 1-39.

[2] Ma, P.C.; Siddiqui, N.A.; Marom, G; Kim, J.K. "Dispersion and functionalization of carbon nanotubes for polymer-based nanocomposites: a review. Composites Part A: Applied Science and Manufacturing 41, no. 10 (2010): 1345-1367.

[3] Liao, Y.; Yu F.; Long, L.; Wei, B.; Lu, L.; Zhang, J. Low-cost and reliable thin film encapsulation for organic light emitting diodes using magnesium fluoride and zinc sulphide. Thin Solid Films 519, no. 7 (2011): 2344-2348.

[4] Ebnalwaled, A.A.; Thabet, A. controlling the optical constants of PVC nanocomposite films for optoelectronic applications. Synthetic Metals 220 (2016): 374-383.

[5] Sehgal, P.; Narula, A.K. Structural, morphological, optical, and electrical transport studies of poly (3-methoxythiophene)/NiO hybrid nanocomposites. Colloid and Polymer Science 293, no. 9 (2015): 2689-2699.

[6] Hussain, A.M.; Neppolian B.; Kim, S.H.; Kim, J.Y.; Choi, H.C.; Lee, K.; Park, S.J.; Heeger A.J. Improved performance of polymer light-emitting diodes with nanocomposites. (2009): 15-20

[7] Karzazi,Y. Organic light emitting diodes: Devices and applications. J. Mater. Environ. Sci 5, no. 1 (2014): 1-12.

[8] Poplavskyy, D; Su, W.; So, F. Bipolar charge transport, injection and trapping studies in a model green-emitting polyfluorene copolymer. Journal of applied physics 98, no. 1 (2005): 014501.

[9] Dinh, N.N.; Chung, D.N.; Thao T.T.; Hui D. Study of nanostructured polymeric composites used for organic light emitting diodes and organic solar cells. Journal of Nanomaterials 2012 (2012): 99 
[10] Eckle, M.; Decher, G. Tuning the performance of layer-by-layer assembled organic light emitting diodes by controlling the position of isolating clay barrier sheets. Nano Letters 1, no. 1 (2001): 45-49.

[11] Zhang, D.; Ryu, K.; Liu X.; Polikarpov, E.; Ly, J.; Tompson, M.E.; Zhou,C. Transparent, conductive and flexible carbon nanotube films and their application in organic light-emitting diodes. Nano letters 6, no. 9 (2006): 1880-1886.

[12] Che, Y.; Chen, H.; Gui, H.; Liu, J.; Liu, B.; Zhou, C.Review of carbon nanotube nanoelectronics and macroelectronics.Semiconductor Science and Technology 29, no. 7 (2014): 073001.

[13] Wang, G.F.; Tao, X.M.; Chen W.; Wang,R.X.; Yang, A. Improvement in performance of organic light-emitting devices by inclusion of multi-wall carbon nanotubes. Journal of luminescence 126, no. 2 (2007): 602-606.

[14] Shao, M.; Garrett, M.P.; Xu, X.; Ivanov, I.N.; Wong,S.S.; Hu, B.; Effects of single walled carbon nanotubes on the electroluminescent performance of organic light-emitting diodes. Organic Electronics 12, no. 6 (2011): 1098-1102.

[15] Qiu, C.;Chen, H.; Xie, J.; Wong, M.; Kwok, H.S. Praseodymium oxide coated anode foorganic light-emitting diode. Applied physics letters 80, no. 19 (2002): 3485-3487.

[16] Kurahatti, R.V.; Surendranathan A.O.; Kori, S.A.;Singh, N.; Ramesh Kumar, A.V.;Srivastava,S. Defence applications of polymer nanocomposites. Defence Science Journal 60, no. 5 (2010).

[17] Jabbour, G.E.; Kippelen, B.; Armstrong, N.R.; Peyghambarian, N. Aluminum based cathode structure for enhanced electron injection in electroluminescent organic devices. Applied Physics Letters 73, no. 9 (1998): 1185-1187.

[18] Jabbour, G. E.; Kawabe, Y.; Shaheen, S. E.; Wang, J. F.; Morrell, M. M.; Kippelen, B.; Peyghambarian,N. Highly efficient and bright organic electroluminescent devices with an aluminum cathode. Applied physics letters 71, no. 13 (1997): 1762-1764.

[19] Yu-Fan Chang, Hsin-Fei Meng, Gang-Lun Fan, Ken-Tsung Wong, Hsiao- 
Wen Zan, Hao-Wu Lin, Heh-Lung Huang, and Sheng-Fu Horng. "Blade coating of Tris (8-hydroxyquinolinato) aluminum as the electron-transport layer for all-solution blue fluorescent organic light-emitting diodes." Organic Electronics29 (2016): 99-106

[20] Kim, H.; Horwitz J. S.; Kim W. H.; Mäkinen A. J.; Kafafi Z. H.; Chrisey D. B. Doped $\mathrm{ZnO}$ thin films as anode materials for organic light-emitting diodes. Thin solid films 420 (2002): 539-543.

[21] Marks, T. J.; Veinot, J. G. C.; Cui J.; Yan H.; Wang A.; Edleman N. L.; Ni J.; Huang Q.; Lee P.; Arm.strong N. R. Progress in high work function TCO OLED anode alternatives and OLED nanopixelation. Synthetic Metals 127, no. 1 (2002): 29-35.

[22] Zhang, T.; Wang, J.; Zhou, M.; Ma, L.; Yin, G.; Chen, G.; Li, Q. Influence of polyhedral oligomeric silsesquioxanes (POSS) on blue light-emitting materials for OLED. Tetrahedron 70, no. 14 (2014): 2478-2486.

[23] Froehlich, J.D.;Young, R.; Nakamura, T.; Ohmori, Y.; Li, S.; Mochizuki, A.;Lauters, M.;Jabbour, G.E. Synthesis of multi-functional POSS emitters for OLED applications. Chemistry of Materials 19, no. 20 (2007): 4991-4997. 